\title{
A Comparison of Methods for Generating Poisson Disk Distributions
}

\author{
Ares Lagae and Philip Dutré
}

Dept. Comp. Sci., Kath. Univ. Leuven

\begin{abstract}
Poisson disk distributions have many applications in the field of computer graphics. Besides sampling, Poisson disk distributions are used in object distribution, non-photorealistic rendering and procedural texturing. Over the years, a large number of methods for generating Poisson disk distributions have been proposed, making it difficult to choose the right method for a given application. In this paper, we present a detailed comparison of most techniques for generating Poisson disk distributions. The methods we study include dart throwing, relaxation dart throwing, Lloyd's relaxation, Shade's Poisson disk tiles, tiled blue noise samples, fast hierarchical importance sampling with blue noise properties, edge-based Poisson disk tiles, template Poisson disk tiles, corner-based Poisson disk tiles and recursive Wang tiles for real-time blue noise. Analysing all of these methods within a single framework is one of the major contributions of this work.
\end{abstract}

Keywords: Poisson disk distributions, spectral analysis

ACM CCS: I.3.3 [Computer Graphics]: Picture/Image Generation

\section{Introduction}

Poisson disk distributions are used frequently in computer graphics. One of the most important applications is sampling. Over the last decades, many different sampling patterns have been studied and analysed. It is now generally accepted that, because of its blue noise properties, the Poisson disk distribution is one of the best sampling patterns for a wide range of applications [Yel82]; [Yel83]; [DW85]; [Coo86]; [Mit87]; [Mit91]. In addition to sampling, Poisson disk distributions have several other important applications in computer graphics, such as object distribution [DHL*98], [CSHD03], primitive distribution for illustration [SHSO2] and texture basis functions [LD05a].

Because of this wide interest, a large number of methods for generating Poisson disk distributions has been developed over the years. These methods, and the distributions they generate, often have very different characteristics. This makes it difficult to choose the right method for a given application. In this paper, we address this problem by comparing the dif- ferent methods and the resulting distributions in detail and providing guidelines to decide which method to use.

This paper is structured as follows. In Section 2, we give an overview of related work. In Section 3, we review techniques for analyzing Poisson disk distributions. In the following sections, we discuss several methods for generating Poisson disk distributions in detail. These include dart throwing (Section 4), relaxation dart throwing (Section 5), Lloyd's relaxation (Section 6), Shade's Poisson disk tiles (Section 7), tiled blue noise samples (Section 8), fast hierarchical importance sampling with blue noise properties (Section 9), edge-based Poisson disk tiles (Section 10), template Poisson disk tiles (Section 11), corner-based Poisson disk tiles (Section 12) and recursive Wang tiles for real-time blue noise (Section 13). In Section 14 we conclude.

The material is presented chronologically. Each method is briefly explained before it is discussed. However, for more complicated methods, the explanation may be a bit compact. We assume the reader is familiar with most methods. 
However, we have added the appropriate references for when this is not the case.

\section{Related Work}

Poisson disk distributions were introduced in the field of computer graphics to solve the aliasing problem. Aliasing is a major source of artifacts in digitally synthesized images. This problem was first identified by Crow [Cro77]. Dippé and Wold [DW85], Cook [Coo86] and Mitchell [Mit87] introduced non-uniform sampling to turn regular aliasing patterns into featureless noise, which is perceptually less objectable. The Poisson disk distribution was identified as one of the best sampling patterns. Their work was based on studies by Yellot [Yel82,Yel83], who found that the photoreceptors in the retina of the eye are distributed according to a Poisson disk distribution, an indication that this sampling pattern is effective for imaging.

Poisson disk distributions are traditionally generated using an expensive dart throwing algorithm [Coo86]. Fast methods that generate approximate Poisson disk distributions have been suggested by various authors [DW85, Mit87, Mit91, Kla00]. The algorithm mostly used nowadays is due to [MF92]. It generalizes over the dart throwing approach, and uses Lloyd's relaxation scheme [Llo82] to optimize the generated distribution.

Already in 1985, Dippé and Wold [DW85] suggested to replicate a pre-computed tile with Poisson disk distributed points across the plane. Since then, several tile-based methods were proposed. Most of them use Wang tiles, square tiles with coloured edges [Wan61, Wan65, GS86, CSHD03]. The first tile based method, an extension of the dart throwing algorithm, was presented by Shade et al. [SCM00]. Hiller et al. [HDK01] used Lloyd's relaxation algorithm to construct a Poisson disk distribution over a set of Wang tiles. This method of was later adopted by Cohen et al. [CSHD03]. Ostromoukhov et al. [ODJ04] presented an interesting technique to generate a distribution with blue noise properties over a given density, based on the Penrose tiles and Lloyd's relaxation scheme. Lagae and Dutré proposed improved construction methods for Poisson disk tiles based on Wang tiles [LD05a] and corner tiles [LD06a], and also studied requirements for generating tiled Poisson disk distributions with good spectral properties [LD05b]. Kopf et al. [KCODL06] presented a method to generate Poisson disk distributions over a given density in real time, based on recursive Wang tiles that contain self-similar and progressive Poisson distributions.

Recently, Jones [Jon06] and Dunbar and Humphreys [DH06] presented efficient implementations of the dart throwing algorithm.

Tools to analyse the spectral properties of point sets were introduced by Ulichney [Uli87], in the context of dithering.

\section{Specification and Analysis}

A Poisson disk distribution is a 2D uniform point distribution in which all points are separated from each other by a minimum distance. Half that distance is called the radius $r$ of the distribution. If a disk of that radius is placed at each point, then no two disks overlap.

\subsection{Radius specification}

To specify the radius of a Poisson disk distribution, we follow the convention of Lagae and Dutré [LD05a]. Rather than using the absolute radius $r$, we use the relative radius $\rho$.

The densest packing of disks in the plane is the hexagonal lattice [Ste99], with a packing density $\eta$ of

$$
\eta=\frac{\pi}{2 \sqrt{3}} \approx 0.9069 \text {. }
$$

The packing density is defined as the fraction of the area filled by the disks. When distributing $N$ disks over a unit toroidal domain, the maximum disk area is thus $\eta / N$. The maximum possible disk radius $r_{\max }$ is therefore given by

$$
r_{\max }=\sqrt{\frac{1}{2 \sqrt{3} N}} .
$$

The Poisson disk radius $r$ of a given distribution of points can be specified as a fraction $\rho \in[0 \ldots 1]$ of the maximum disk radius

$$
r=\rho r_{\max }
$$

Contrary to the absolute radius, the relative radius is independent of the number of points and the size of the domain of the point distribution. The relative radius is a measure of how well the points are distributed. A uniformly distributed point set can have a relative radius of 0 , and the relative radius of a hexagonal lattice equals 1 . Poisson disk distributions should have a relative radius that is large ( $\rho \geq 0.65)$, but not too large ( $\rho \leq 0.85$ ), because regular configurations must be avoided.

\subsection{Spectral analysis}

Radial statistics are the first important tool to evaluate the quality of a Poisson disk distribution. A second important tool is a study of the frequency domain characteristics of the Poisson disk distribution. The technique described here was first used by Ulichney to study dither patterns [Uli87].

To estimate the power spectrum $P(f)$, Ulichney uses Bartlett's method of averaging periodograms [Bar55]. The periodogram of a Poisson disk distribution of $N$ points $\left\{x_{0}, \ldots, x_{N-1}\right\} \subset[0,1)^{2}$ is the magnitude squared of the Fourier transform of the distribution

$$
\left|\mathcal{F} \frac{1}{N} \sum_{j=0}^{N-1} \delta\left(x-x_{j}\right)\right|^{2},
$$




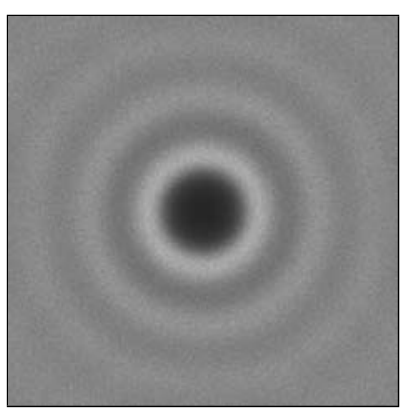

(a)



(b)

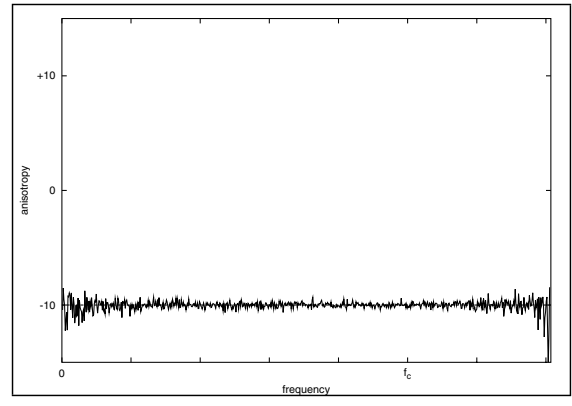

(c)

Figure 1: The typical (a) power spectrum, (b) radially averaged power spectrum and (c) anisotropy of Poisson disk distributions.

where $\mathcal{F}$ denotes the Fourier transform and $\delta$ is Dirac's delta function. By averaging $K$ periodograms, an estimate of the power spectrum $\hat{P}(f)$ is obtained with variance

$$
\operatorname{var}\{\hat{P}(f)\} \approx \frac{1}{K} P^{2}(f) \text {. }
$$

Thus, the spectral properties of a method for generating Poisson disk distributions are obtained by averaging periodograms from Poisson disk distributions generated with that method. It is important to note that a periodogram is associated with a single Poisson disk distribution, while the power spectrum estimate is associated with a specific method for generating Poisson disk distributions (e.g. a specific algorithm with a fixed set of parameters).

The periodogram of a Poisson disk distribution is radially symmetric. Therefore, two one-dimensional statistics are derived from the power spectrum. The first one is the radially averaged power spectrum

$$
P_{r}\left(f_{r}\right)=\frac{1}{N_{r}\left(f_{r}\right)} \sum_{i=1}^{N_{r}\left(f_{r}\right)} \hat{P}(f),
$$

which is obtained by averaging $\hat{P}(f)$ in concentric annuli of width $\Delta$. Each annulus has a central radius $f_{r}$ and contains $N_{r}\left(f_{r}\right)$ frequency samples. The second statistic is the anisotropy

$$
A_{r}\left(f_{r}\right)=\frac{s^{2}\left(f_{r}\right)}{P_{r}^{2}\left(f_{r}\right)}
$$

where the sample variance of the frequency samples is defined as

$$
s^{2}\left(f_{r}\right)=\frac{1}{N_{r}\left(f_{r}\right)-1} \sum_{i=1}^{N_{r}\left(f_{r}\right)}\left(\hat{P}(f)-P_{r}\left(f_{r}\right)\right)^{2} .
$$

The anisotropy is a measure for the radial symmetry of the power spectrum.

Figure 1 shows the typical power spectrum of a Poisson disk distribution. The corresponding radially averaged power spectrum and anisotropy are also shown. The power spectrum reveals the typical blue noise properties. The central DC peak is surrounded by an annulus of low energy, followed by a sharp transition region, a low-frequency cutoff at the principal frequency $1 / 2 r$, and a flatter high-frequency region.

Figure 2 shows the typical power spectrum and radially averaged power spectrum and anisotropy of a uniform distribution. As expected, the power spectrum is flat.

In this work, we judge the quality of methods for generating Poisson disk distributions by comparing radial statistics and power spectra. These two measures cover a wide range of applications. Other measures exist, but these are usually geared towards a specific application (e.g. numerical integration).

As in Ulichney's work, the spectral estimates in this paper were produced by averaging $K=10$ periodograms. Therefore, an anisotropy of $-10 d B$ should be considered background noise. In both cases, the anisotropy is low (close to $-10 d B$ ), indicating good radial symmetry. A reference line at $-10 d B$ appears in all anisotropy plots.

All spectral estimates in this paper were obtained by analyzing distributions of 16384 (or as close as possible 16384) points. The periodogram resolution used to analyse a distribution of $N$ points is determined as

$$
\frac{1}{0.75 r_{\max }} \text {. }
$$

This ensures that the periodogram covers a domain corresponding to twice the principal frequency of a Poisson disk distribution with a relative radius of $0.75(634 \times 634$ for 16384 points). This number is rounded to the next power of two $(1024 \times 1024$ for 16384 points). Thus, all power spectra and plots are at the same scale. The width of the annuli $\Delta$ is one sample.

All power spectrum images were tone mapped with a logarithmic tone mapper, using the same settings for all images. The radially averaged power spectrum was normalized. The high-magnitude DC peak was removed from all plots. 


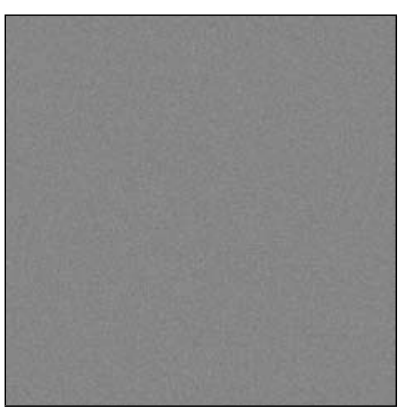

(a)



(b)

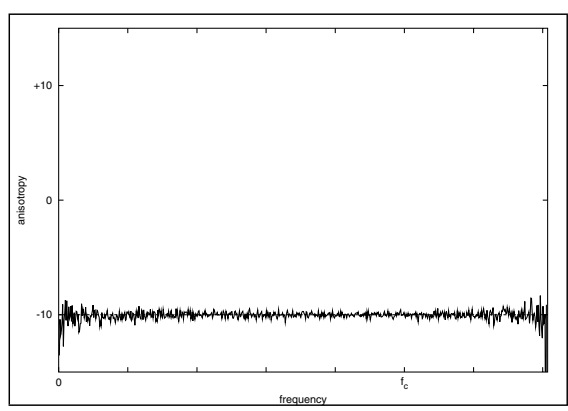

(c)

Figure 2: The typical (a) power spectrum, $(b)$ radially averaged power spectrum and $(c)$ anisotropy of uniform distributions.

Please note that comparing periodograms, power spectrum estimates and radially averaged power and anisotropy graphs of previously published papers is very difficult. Often the distinction between periodogram and power spectrum is not made, periodograms and power spectra are tone mapped with different settings, and power and anisotropy graphs are computed with wide annuli, effectively smoothing the graphs. Analysing all methods within a single framework is the major contribution of this paper.

\subsection{Timings}

For most methods discussed in this paper we have included timings, to give the reader a rough idea of the relative computational cost of the different techniques. The timings were obtained on a regular desktop PC with an AMD Athlon(tm) 64 Processor $4000+\mathrm{CPU}$ running at $2.4 \mathrm{GHz}$. Where available, we used example code provided by the authors of the techniques, so please note that the timings are only indicative.

\section{Dart Throwing}

In the mid-80s, Dippé and Wold [DW85], Cook [Coo86] and Mitchell [Mit87] introduced non-uniform sampling and the Poisson disk distribution to solve the aliasing problem. Cook [Coo86] proposed the dart throwing algorithm for generating Poisson disk distributions.

The dart throwing algorithm generates uniformly distributed points, and rejects points that do not satisfy the minimum separation with already generated points. This process continues until no more points can be added. To correctly handle boundary conditions, the Poisson disk distributions are typically generated over a toroidal domain.

Dart throwing is widely used and is easy to implement. However, the algorithm is also slow, and difficult to control: instead of specifying the number of points, the radius of the distribution has to be provided, the final number of points
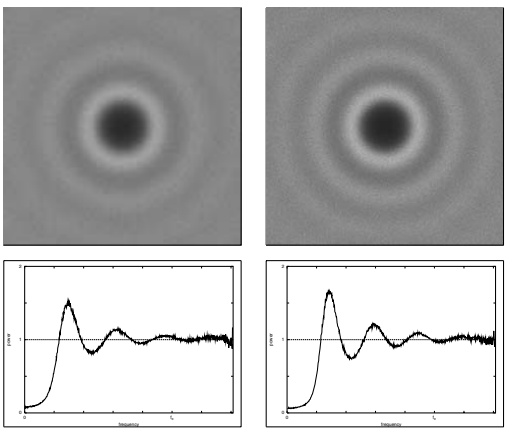



(a)

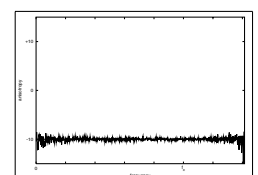

(b)
Figure 3: The power spectrum of Poisson disk distributions generated with the dart throwing algorithm, with a relative radius of (a) 0.70 and (b) 0.75 .

in the distribution is difficult to predict, and if the process is stopped too soon, the density of the points is not uniform.

The dart throwing algorithm can be improved by combining it with the relative radius specification. The improved algorithm takes as input the desired number of points and the relative radius of the Poisson disk distribution. This solves the radius specification problem. Experiments show that dart throwing is capable of generating Poisson disk distributions with a relative radius up to 0.75 . Termination, however, is still not guaranteed, and generating large Poisson disk distributions $(100,000$ points or more $)$ is very difficult in practice.

Figure 3 shows the power spectrum of Poisson disk distributions generated with the dart throwing algorithm. The radially averaged power spectrum exhibits the typical blue noise properties, and the anisotropy is low. 

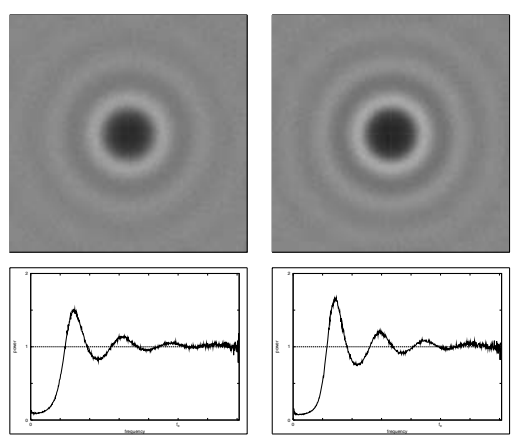

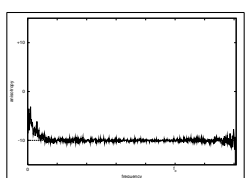

(a)

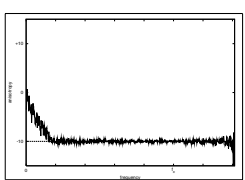

(b)


(a)
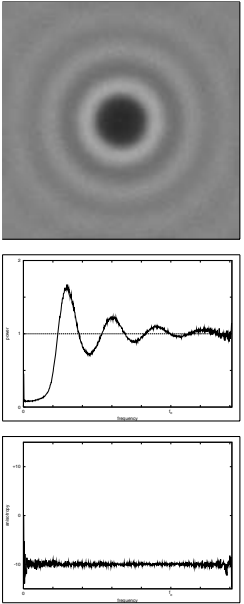

(b)
Figure 4: The power spectrum of Poisson disk distributions generated with the accelerated dart throwing algorithm of Jones, with a relative radius of (a) 0.70 and (b) 0.75 .

The time required to generate a Poisson disk distribution of 16384 points with a relative radius of 0.70 and 0.75 is, respectively, 34.997 and 263.492 seconds.

Although the traditional dart throwing algorithm is terribly inefficient, it is the most natural way for generating a Poisson disk distribution. Therefore, we will use the power spectra of Figure 3 as a reference.

Recently, Jones [Jon06] presented an efficient implementation of the dart throwing algorithm, based on the Voronoi diagram. Figure 4 shows the power spectrum of Poisson disk distributions generated with this method. As expected, the method has the same spectral properties as the traditional dart throwing algorithm. However, the generated distributions are not toroidal. This explains the central horizontal and vertical lines in the power spectrum, and the increased anisotropy at low radial frequencies. The algorithm of Jones is very fast. The time required to generate a Poisson disk distribution of 16384 points with a relative radius of 0.70 and 0.75 is respectively 3.511 and 3.403 seconds.

Dunbar and Humphreys [DH06] recently also presented efficient implementations of the dart throwing algorithm, based on a data structure using scalloped sectors. Figure 5 shows the power spectrum of Poisson disk distributions generated with their logarithmic algorithm. As expected, the method has the same spectral properties as the traditional dart throwing algorithm. Next to the logarithmic algorithm, Dunbar and Humphreys also present a linear and boundary sampling algorithm. The power spectrum of those methods is shown in Figure 6. The linear method is supposed to be slightly faster than the logarithmic algorithm. The peaks in the power
Figure 5: The power spectrum of Poisson disk distributions generated with the accelerated dart throwing algorithm of Dunbar and Humphreys, with the same radius as in Figure 3.


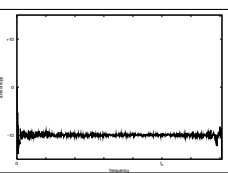

(a)

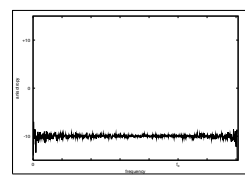

(b)

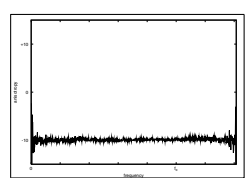

(c)
Figure 6: The power spectrum of Poisson disk distributions generated with the (a) logarithmic, (b) linear and (c) boundary sampling algorithm of Dunbar and Humphreys.

spectrum at multiples of the principal frequency are slightly larger and seem to be a bit skew. The boundary sampling method is significantly faster than the other two algorithms. The peaks in the power spectrum at multiples of the principal frequency are significantly larger than the reference power spectrum. The time required to generate a Poisson disk distribution with an absolute radius of 0.005877 (corresponding with a relative radius of 0.70 in the previous methods) using 



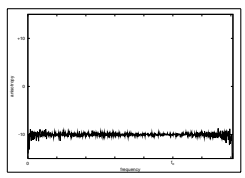

(a)

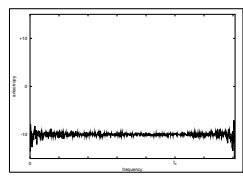

(b)
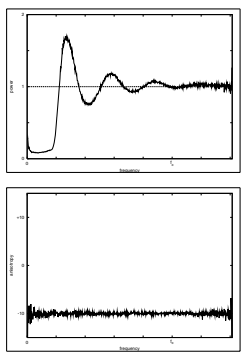

(c)
Figure 7: The power spectrum of Poisson disk distributions generated with relaxation dart throwing. The Poisson disk distributions were generated with an initial radius 0.15 , and the radius was reduced by a factor of $(a) 0.9,(b) 0.99$ and (c) 0.999 after (a) 100, (b) 1000 and (c) 10000 failed attempts. The relative radius of the final Poisson disk distributions is approximately (a) $0.57,(b) 0.68$ and (b) 0.73 .

the logarithmic, linear and boundary algorithm is respectively 7.002, 7.911 and 0.137 seconds.

\section{Relaxation Dart Throwing}

In 1992, McCool and Fiumé [MF92] proposed an improved version of the dart throwing algorithm, which we call relaxation dart throwing.

Relaxation dart throwing is similar to dart throwing. However, points are placed with a large radius initially, and once no more space has been found for a large number of attempts, the radius is reduced by some fraction. The Poisson disk distributions generated with relaxation dart throwing are usually toroidal, and also hierarchical.

This algorithm has several advantages compared to dart throwing: it is somewhat faster, it allows to specify the desired number of points rather than the radius, and termination is guaranteed.

Figure 7 shows the power spectrum of Poisson disk distributions generated with the relaxation dart throwing. The spectral characteristics of Poisson disk distributions generated with dart throwing and relaxation dart throwing are very similar. The transition region seems to be a little bit steeper for Poisson disk distributions generated with relaxation dart throwing.

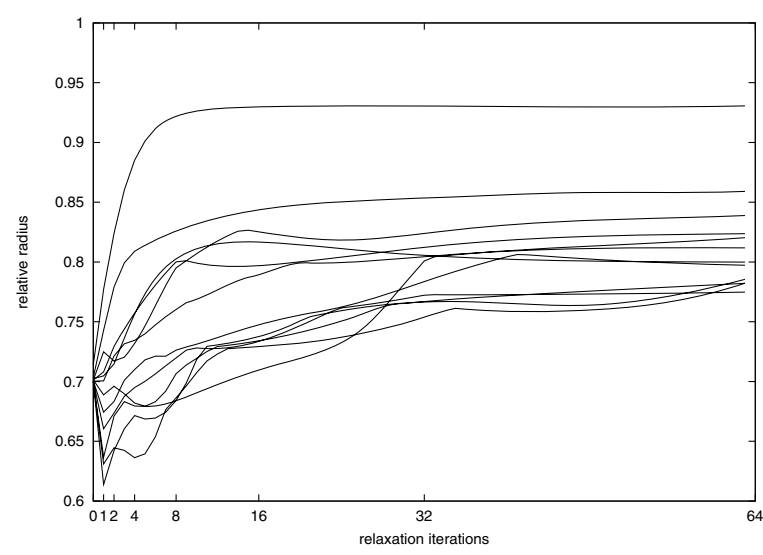

Figure 8: The relative radius of Poisson disk distributions of 8 (top), 16, 32, 64, 128, 256, 512, 1024, 2048, 4096, 8192 and 16384 (bottom) points, generated with dart throwing, versus the number of relaxation iterations.

The time required to generate a Poisson disk distributions of 16384 points using an initial radius 0.15 , a radius reduction factor of 0.9, 0.99 and 0.999 and 100, 1000 and 10,000 failed attempts is, respectively, $0.020,0.289$ and 11.733 seconds. The relative radius of the generated distributions is respectively, $0.57,0.68$ and 0.73 .

\section{Lloyd's Relaxation}

After a Poisson disk distribution is generated using relaxation dart throwing, McCool and Fiumé [MF92] apply Lloyd's relaxation [Llo82] to increase the radius of the Poisson disk distribution.

Lloyd's relaxation is an iterative process: in each iteration, the Voronoi diagram of the point set is computed, and each point is moved to the centroid of its Voronoi cell. To correctly handle boundary conditions, Lloyd's relaxation is performed over a toroidal domain.

Lloyd's relaxation can be used as a post-process for increasing the radius of a Poisson disk distribution generated with any technique, or as a standalone technique that generates a Poisson disk distribution from an uniform point distribution.

Figure 8 shows the radius of Poisson disk distributions generated with dart throwing versus the number of relaxation iterations. For distributions up to 16384 points, Lloyd's relaxation converges in about 16 to 32 iterations. Poisson disk distributions with less points seem to converge to a slightly higher radius, but in general all distributions settle for a relative radius of about 0.75 to 0.85 . Lloyd's relaxation is the only technique that allows to generate Poisson disk distributions with such a high radius. 





(a)
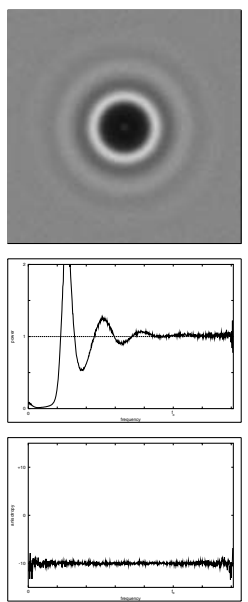

(d)
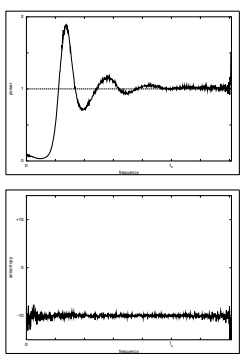

(b)
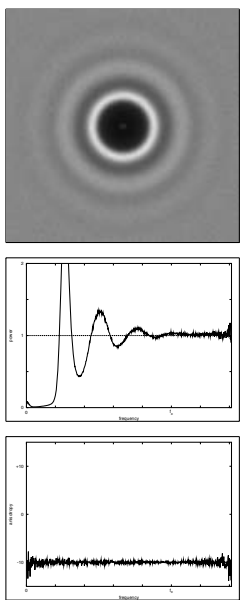

(e)
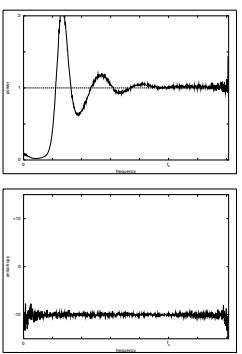

(c)
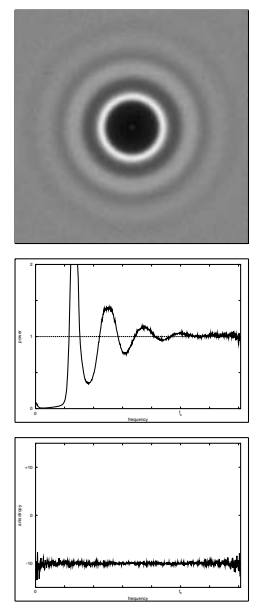

(f)
Figure 9: The power spectrum of Poisson disk distributions generated with dart throwing after (a) $0,(b) 1,(c) 2,(d) 4$, (e) 8 and $(f) 16$ relaxation iterations.

Figure 9 shows the power spectrum of Poisson disk distributions generated with dart throwing after several relaxation iterations. The peak at the principal frequency, and the smaller peaks at multiples of the principal frequency, increase in magnitude with each iteration. The transition region becomes steeper with each iteration.

Hiller et al. [HDK01] report that Lloyd's relaxation results in an increased anisotropy, but the anisotropy graphs do not support that claim, or the increase is not significant. It is possible that Lloyd's relaxation, after many iterations, finds the global minimum (a hexagonal grid), and that the Poisson disk distribution regains periodicity. We have seen this happening for smaller distributions, but for larger distributions this does not seem to be a problem. Also, the problem can be avoided by monitoring the radius during relaxation.

The time required for 1, 2, 4, 8, 16 and 32 iterations, starting from a uniform distribution of 16384 points, is respectively, $21.308,35.661,64.397,120.448,234.140$ and 458.460 seconds.

\section{Shade's Poisson Disk Tiles}

In 2000, Shade et al. [SCM00] presented the first tile-based approach for generating Poisson disk distributions. Their approach is an extension of the dart throwing algorithm and uses Wang tiles [Wan61, Wan65, GS86, CSHD03].

Wang tiles are square tiles with coloured edges. To tile the plane with a set of Wang tiles, the tiles are placed with their corners on the integer lattice points, such that adjoining edges have matching colors. The tiles cannot be rotated.

Shade et al. construct a Poisson disk distribution over a set of eight Wang tiles using an algorithm similar to dart throwing. Before a point is added to a tile, all possible neighbouring tiles are checked, and the point is rejected if the minimum separation criterion is not met. Once a Poisson disk distribution is constructed over a set of Wang tiles, arbitrary large Poisson disk distributions can be generated in real time by stochastically tiling the plane with the Wang tiles. For more details, we refer to [SCM00].

However, as noted by Cohen et al. [CSHD03] and Lagae and Dutré [LD05a], this approach is flawed. The constraints of multiple tiles cause less points to be inserted near the edges and corners. This results in a noticeable lower density of points in those regions. The problem of multiple constraints is more severe than it might seem at first sight: placing a point near the corner of one tile makes it impossible, for at least one corner of every other tile in the tile set, to have a point nearby.

Although these problems limit the applicability of this method, the idea of using Wang tiles for generating Poisson disk distributions would later prove to be very valuable.

\section{Tiled Blue Noise Samples}

In 2001, Hiller et al. [HDK01] presented an approach based on Lloyd's relaxation algorithm rather than dart throwing to construct a Poisson disk distribution over a set of Wang tiles.

Hiller et al. use a set of eight Wang tiles. An initial point set is generated in the center of every tile. Each tile in the set is surrounded by all possible configurations of 8 tiles. For all of these configurations, a Voronoi diagram is constructed. Each Voronoi diagram determines a displacement vector for 


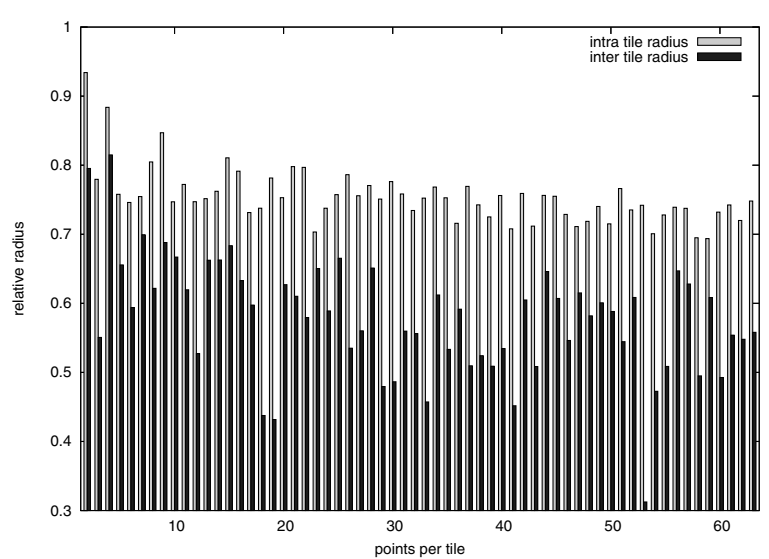

Figure 10: The intra and inter tile radius of the Poisson disk tile sets of Hiller et al.

every point in the tile. All displacement vectors are averaged, and the points in the tile are moved accordingly. This process is iterated, until the point distributions stabilize. For more details, we refer to [HDK01].

To analyse this method, we reviewed the original data of Hiller et al. The data consists of 63 tile sets, with 1 to 63 points per tile. For each of these tile sets, the positions of the points from iteration 0 to 29 are given.

From the original data we computed radial statistics, which were not listed in the original paper. Figure 10 shows the intraand inter-tile radius of the 63 tile sets. The intra-tile radius, which is the radius of the Poisson disk distribution within a single tile, is relatively high ( $\rho \approx 0.80$ ), as expected from an approach based on Lloyd's relaxation (Section 6). However, the inter-tile radius, which is the radius of the Poisson disk distribution taking into account neighbouring tiles, and thus the final radius of the generated distribution, is relatively low (often $\rho \approx 0.40$ or even less). This shows that the low radius is due to difficulties at the tile edges, which indicates problems with the construction method. Figure 11 shows the intra and inter tile radius versus the number of relaxation iterations for two tile sets. As expected, the intra-tile radius converges to an relative radius of about 0.80 . The inter-tile radius, however, does not seem to converge.

Figure 12 shows power spectra of Poisson disk distributions generated with the method of Hiller et al. Compared to the previous methods we have discussed, the power spectra are relatively bad. In Section 11, however, we will see that eight tiles is not enough for generating Poisson disk distributions with good spectral properties. Furthermore, the algorithm most likely is not capable of handling larger tile sets. Even with eight tiles, the number of eight-tile configurations is so large that the displacement vectors tend to average each other out.



(a)



(b)

Figure 11: The intra and inter tile radius versus the number of relaxation iterations for the Poisson disk tile set of Hiller et al. with (a) 19 and (b) 53 points per tile.

We also suspect that due to the construction method and convergence problems, the point density along the tile edges is lower than in the center of the tiles, but we have no hard evidence supporting this claim.

The method of Hiller et al. was later adopted in the paper of Cohen et al. [CSHD03], a paper that popularized Wang tiles in the field of computer graphics.

\section{Fast Hierarchical Importance Sampling with Blue Noise Properties}

In 2004, Ostromoukhov et al. [ODJ04] presented an interesting technique for generating point distributions with blue noise properties over a given density.

The points are placed on the vertices of a Penrose tiling [Pen74, GS86], recursively subdivided according to a given density. The point distribution is optimized by moving the points according to pre-computed displacement vectors 



(a)
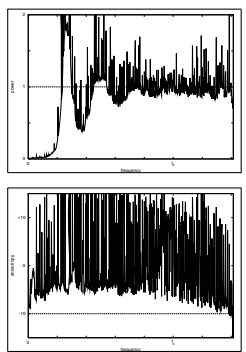

(b)
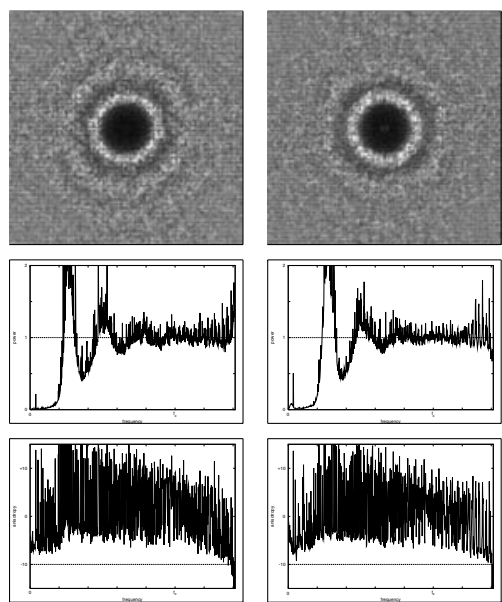

(d)
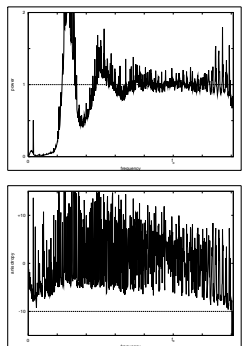

(e)
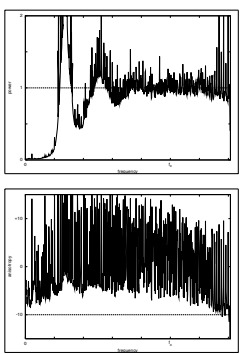

(c)
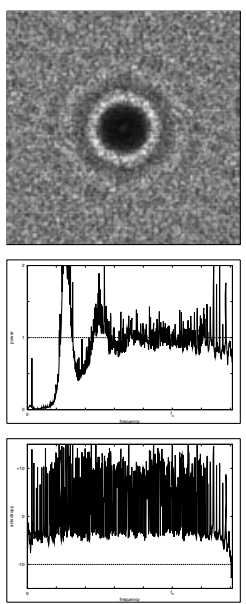

(f)

Figure 12: The power spectrum of Poisson disk distributions generated with the Poisson disk tile sets of Hiller et al. with (a) 16, (b) 19, (c) 32, (d) 41, (e) 53 and (f) 63 points per tile.

obtained with Lloyd's relaxation. For more details, we refer to [ODJ04].

The radius of the point distributions generated over a constant density is comparable with dart throwing. The relative radii we have measured varied between 0.65 and 0.75 .

Because multiple runs of the code produce the same point distribution, it is not possible to compute a power spectrum estimate. Instead, Figure 13 shows the periodogram of a Poisson disk distribution generated with Ostromoukhov's approach. For comparison, the periodogram of a Poisson disk distribution generated with edge-based Poisson disk tiles (Section 10) and corner-based Poisson disk tiles (Section 12) is included. The periodogram of Ostromoukhov's approach shows severe
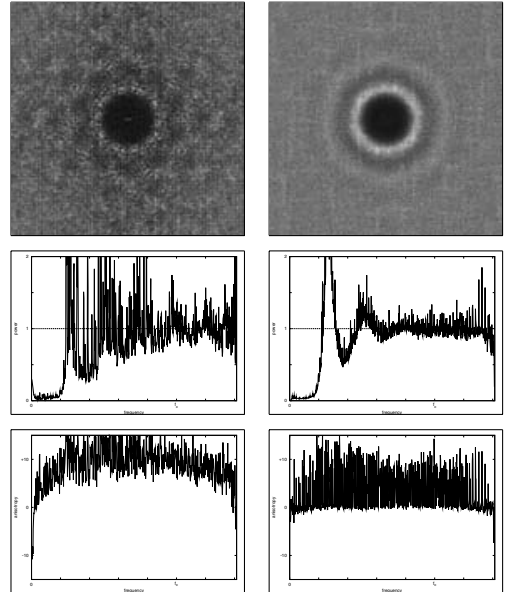

(a)

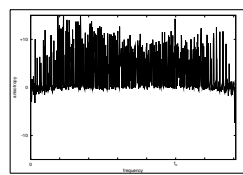

(b) reference
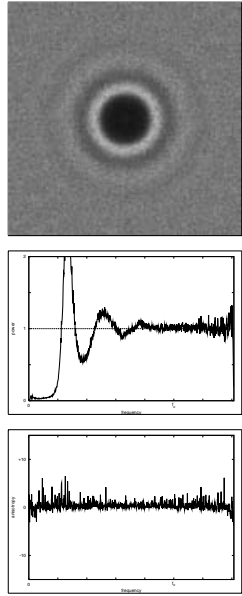

(c) reference
Figure 13: The periodogram of (a) a Poisson disk distribution generated with the approach of Ostromoukhov et al., (b) a Poisson disk distribution generated with edge-based Poisson disk tiles with 64 points per tiles, and a relative radius of 0.75 and (c) a Poisson disk distribution generated with corner-based Poisson disk tiles with six colours, 64 points per tiles, and a relative radius of 0.75 .

artefacts. A star-like pattern of large spikes revealing the 10 -fold symmetry of the underlying Penrose tiling is visible. The anisotropy of Ostromoukhov's approach also is significantly higher.

The time needed to generate a Poisson disk distribution of 16384 points is 0.192 seconds.

\section{Edge-Based Poisson Disk Tiles}

In 2005, Lagae and Dutré [LD05a] presented a procedural object distribution function, a new texture-basis function. This application required Poisson disk distribution with a large radius, meaning the approach of Hiller et al. could not be used. Lagae and Dutré presented a new kind of Poisson disk tiles which they call edge based Poisson disk tiles.

Edge-based Poisson disk tiles are constructed over a complete set of Wang tiles. A complete set of Wang tiles contains all possible combinations of edge colours. A complete Wang tile set over $C$ colours consists of $C^{4}$ tiles.

The construction method follows from a study of the different regions in a tile. Three kinds of regions in a tile are identified: edge regions, corner regions and an interior region.

These regions determine a dual tiling. The dual tiling uses three kinds of tiles: edge tiles, which correspond to the union of edge regions of neighboring Wang tiles corner tiles, which 


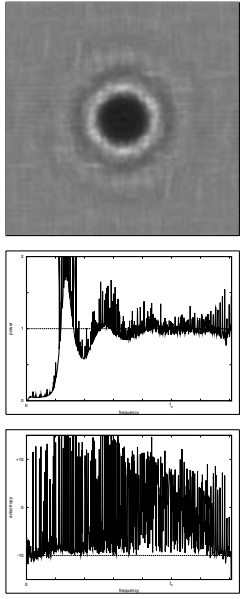

(a)
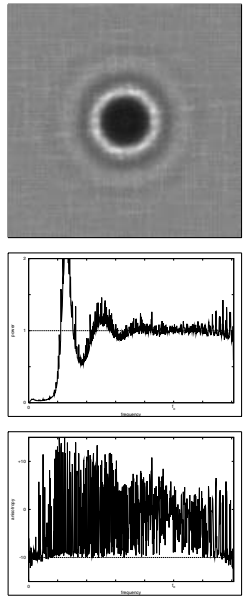

(b)


(c)
Figure 14: The power spectrum of Poisson disk distributions generated with edge-based Poisson disk tiles, with a relative radius of 0.75 and (a) 32, (b) 48 and (c) 64 points per tile.

correspond to the union of the corner regions of four neighboring Wang tiles, and interior tiles, which correspond to the interior region of the Wang tiles.

For a complete set of Wang tiles over $C$ colours, there are $C$ horizontal and $C$ vertical edge tiles, one for each colours, $C^{4}$ corner tiles because a corner tile is determined by four edge tiles, and $C^{12}$ interior tiles, because an interior tile is determined by four corner tiles or 12 edges.

Poisson disk distributions are generated over, respectively, the edge tiles, the corner tiles and the interior tiles. Then the Poisson disk tiles are constructed from parts of these tiles.

A Poisson disk tile set based on a complete Wang tile set over $C$ colour, will contain $C^{12}$ tiles. For $C=2$, this comes down to 4096 tiles. This is the only practical choice for the number of colour, as $C=1$ results in a single toroidal tile, and $C=3$ produces over half a million tiles. Note that, the Poisson disk tile set does not contain $C^{4}$ tiles. This is because corner tiles take into account the colours of all their incident edge tiles. For more details, we refer to [LD05a].

Figures 14 shows power spectra of Poisson disk distributions generated with edge-based Poisson disk tiles. In contrast with previous methods, the relative radius of the Poisson disk distributions generated with this approach is high (a relative radius up to 0.85). Compared to the previous methods we discussed, the power spectra are relatively good. The power spectra are closer to the reference power spectra, and the magnitude of the peaks is smaller than those of template Poisson disk tiles (Section 11) and tiled blue noise samples (Section 8). This is because more tiles are used and because the tiles do not have as many points in common as template Poisson disk tiles (Section 8). However, there are some strange wavelike artefacts in the spectra that we cannot explain. In Section 11 however, we will see that 4096 tiles is enough but way too much for generating Poisson disk distributions with good spectral properties.

\section{Template Poisson Disk Tiles}

In 2005, Lagae and Dutré [LD05b] also presented template Poisson disk tiles, a very simple tile-based approach. A set of template Poisson disk tiles is a set of square tiles that share the same toroidal boundary of points. Once a set of template Poisson disk tiles is constructed, a tiled Poisson disk distribution can be generated by placing tiles chosen randomly from the tile set next to each other.

A template Poisson disk tile set is constructed by choosing the size of the tile set, the number of points per tile, and the radius of the Poisson disk distribution.

A single tile, called the master tile, is created by generating a toroidal Poisson disk distribution using relaxation dart throwing optionally followed by Lloyd's relaxation. This process is repeated if the desired Poisson disk radius is not reached.

A tiled Poisson disk distribution can be generated using only the master tile, because the distribution it contains is toroidal. However, there will be much repetition in the distribution, something that should be avoided.

To ensure that a Poisson disk distribution is continuous over tile edges, only points closer to an edge of the tile than the Poisson disk radius need to be considered. Thus, a single tile template can be created by discarding all points in the interior of the master tile.

The remaining tiles in the tile set are created by filling the tile template with a Poisson disk distribution that brings the total number of points in the tile to the chosen number of points per tile. Again, this distribution is generated using relaxation dart throwing optionally followed by Lloyd's relaxation. However, no new points are added to the tile template, and during relaxation, points are prohibited to enter the tile template. This is done by clipping displacement vectors of points that are about to enter the tile template. For more details, we refer to [LD05b].

The major advantages of template Poisson disk tiles is that there are no constraints on the size of the tile set and the number of points per tile. This allows to investigate the effect of these parameters on the spectrum of the tiled Poisson disk distributions. Figure 15 shows the power spectrum of tiled Poisson disk distributions generated with template Poisson disk tile sets, for a variety of parameters. In general, the spectra for 16, 24 and 32 points per tile and the spectra 


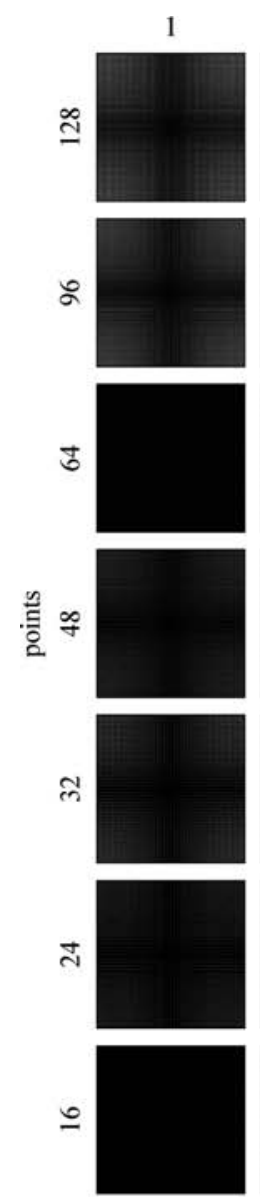

1
2


2
4


4
8


8
16
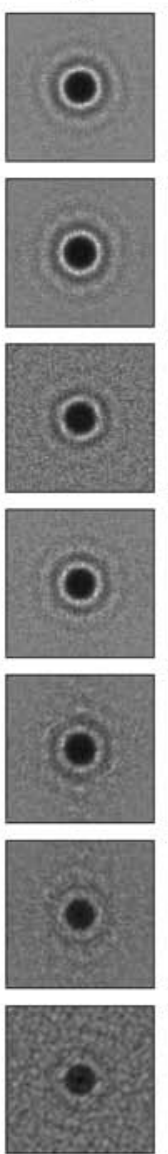

16

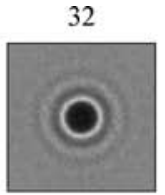

64
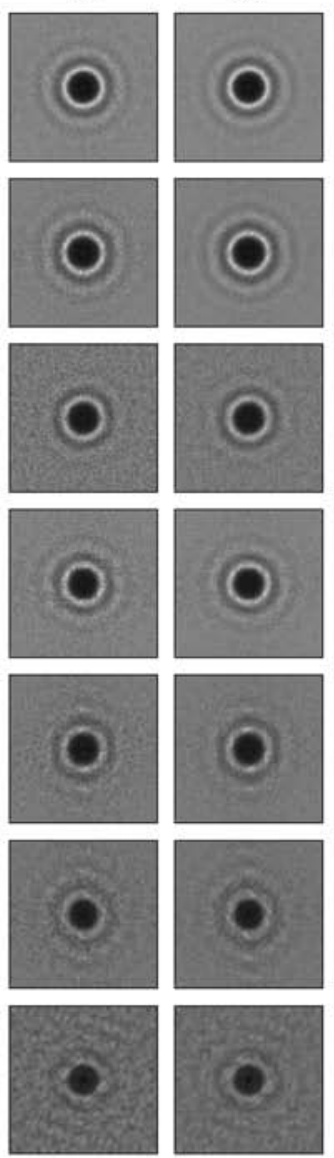

32
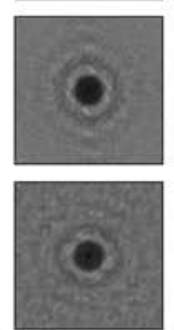

64


128

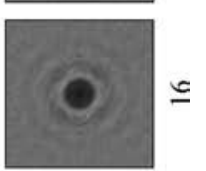

256

Figure 15: The power spectrum of Poisson disk distributions generated with template Poisson disk tiles with a relative radius of 0.75 for template Poisson disk tile sets with 1,2,4,8,16,32,64,128 and 256 tiles and 16,24,32,48,64,96 and 128 points per tile.

for 1, 2, 4, 8, 16 and 32 tiles are rather bad, while spectra for a larger tile set size and number of points per tile are rather good. Therefore it is recommended to use at least 32 or more points per tile and 32 or more tiles. We believe these results generalize to other kinds of Poisson disk tiles, and explain why the power spectrum of tiled blue noise samples (Section 8 ) is rather bad.

Figure 16 shows more detailed power spectra of tiled Poisson disk distributions generated with template Poisson disk tiles. The major difference between the spectrum of a tiled Poisson disk distribution and a non-tiled Poisson disk distribution is that the spectrum of a tiled Poisson disk distribution contains a grid-like pattern of spikes. This effect is most easily explained in terms of autocorrelation. The autocorrelation is related to the power spectrum through the Wiener-Khinchin Theorem. Because only a limited number of tiles are used, the autocorrelation peaks for lags that are a multiple of the tile size. In the power spectrum, this results in a grid-like pattern of peaks on frequencies that are a multiple of the reciprocal of the tile size. When using more tiles, the spikes will decrease in magnitude. However, because only a finite number of tiles is used, it is impossible to eliminate them completely. Note that this effect is not limited to template Poisson disk tiles. The grid is visible in all methods that are based on a square tessellation, including tiled blue noise samples (Section 8), template Poisson disk tiles (Section 11), edge-based Poisson disk tiles (Section 10), corner-based Poisson disk tiles (Section 12), and recursive Wang tiles (Section 13). For template Poisson disk tiles, another factor that contributes to the peaks is the fact that all tiles in the tile set have a number of points in common, namely the points in the tile template, which are replicated periodically when generating a tiled Poisson disk distribution. These peaks also turn up in the radially averaged power spectrum and anisotropy graphs. 

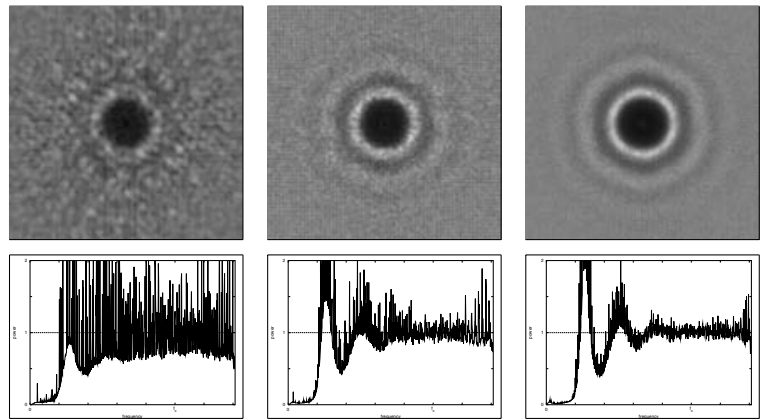



(a)



(b)


(c)
Figure 16: The power spectrum of Poisson disk distributions generated with template Poisson disk tiles with (a) Eight tiles and 24 points per tile, (b) 32 tiles and 48 points per tile, and (c) 128 tiles and 96 points per tile.

Template Poisson disk tiles may not be suited for use in applications. However, they allow to study the effect of the size of the tile set and the number of points per tile on the power spectrum, and help to better understand the spectral characteristics of other tile based approaches.

\section{Corner-Based Poisson Disk Tiles}

In 2006, Lagae and Dutré [LD06a] presented an improved version of their edge-based Poisson disk tiles, which we call corner based Poisson disk tiles.

Corner-based Poisson disk tiles are constructed over a complete set of corner tiles rather than Wang tiles. Corner tiles are square tiles with coloured corners, similar to Wang tiles. The tile construction method is very similar to edgebased Poisson disk tiles. For a set of tiles with coloured corners over $C$ colours, there are $C$ corner tiles, one for each colour, and $C^{2}$ horizontal and vertical edge tiles, because an edge tile is determined by two corner tiles. Now, the corner tiles rather than the edge tiles are constructed first. That is why the tiles are called corner-based Poisson disk tiles. For more details, we refer to [LD06a].

In contrast with edge-based Poisson disk tiles, cornerbased Poisson disk tile sets can be constructed for a variety of tile set sizes. For $C$ colours, an edge-based Poisson disk tile set contains $C^{12}$ tiles. The only practical choice for $C$ is 2, which results in 4096 tiles. For $C$ colours, a corner-based Poisson disk tile set counts only $C^{4}$ tiles, enabling tile sets of 16, 81, 256, 625, 1296, 2401 and 4096 tiles. This solves the problem of edge-based Poisson disk tiles having too many tiles (see Section 10 and Section 11), and also allows trading spectral quality for tile set size.

Figure 17 shows the power spectrum of tiled Poisson disk distributions generated with corner-based Poisson disk tile sets, for a variety of parameters. These results confirm earlier findings (Section 11). Starting from 3 colours (81 tiles) and 32 points per tile the spectra are rather good. The power spectra of corner-based Poisson disk tiles are better than the spectra of edge based Poisson disk tiles (Section 10), even when using smaller tile sets.

Figures 18 shows that the peaks in power spectra generated with corner-based Poisson disk tiles are much smaller than those of edge-based Poisson disk tiles. The radially averaged power spectrum is almost the same as that of non-tiled Poisson disk distributions. Although the anisotropy still contains peaks, it is much smaller than the anisotropy of other Poisson disk tiles.

There is an additional argument to choose corner-based Poisson disk tiles over edge-based Poisson disk tiles. Figure 19 plots the expected number of points in edge and corner regions. The expected number of points in corner regions is constant, and much smaller than the expected number of points in edge regions. Therefore, more variation in edge tiles will produce a better power spectrum than more variation in corner tiles. For $C=2$ colours, a set of edge-based Poisson disk tiles consists of 4096 tiles and there are two different edge tiles (and 16 different corner tiles). For $C=8$ colours, a set of corner-based Poisson disk tiles also counts 4096 tiles, but there are 64 different edge tiles (and eight different corner tiles). This is an important reason why corner based Poisson disk tiles produce better power spectra than edge based Poisson disk tiles.

The time required to generate Poisson disk distributions of approximately 16348 points using a tile set with four colours and 32, 48 and 64 points per tile is, respectively, $0.088,0.102$ and 0.122 seconds. With six colours the time is respectively $0.238,0.319$ and 0.415 seconds, and with eight colours, the time is respectively $0.629,0.911$ and 1.200 seconds. Most of this time is used for loading the tile set stored in a text file in memory.

\section{Recursive Wang Tiles for Real-Time Blue Noise}

In 2006, Kopf et al. [KCODL06] presented a method to generate Poisson disk distributions over a given density in real time.

Kopf et al. use recursive Wang tiles that contain self-similar and progressive Poisson disk distributions. The property of self-similarity allows to increase the point density in large steps by recursively subdividing the tile. A progressive point distribution allows to smoothly adjust the density of points. Combined, these two properties enable an algorithm for gen- 


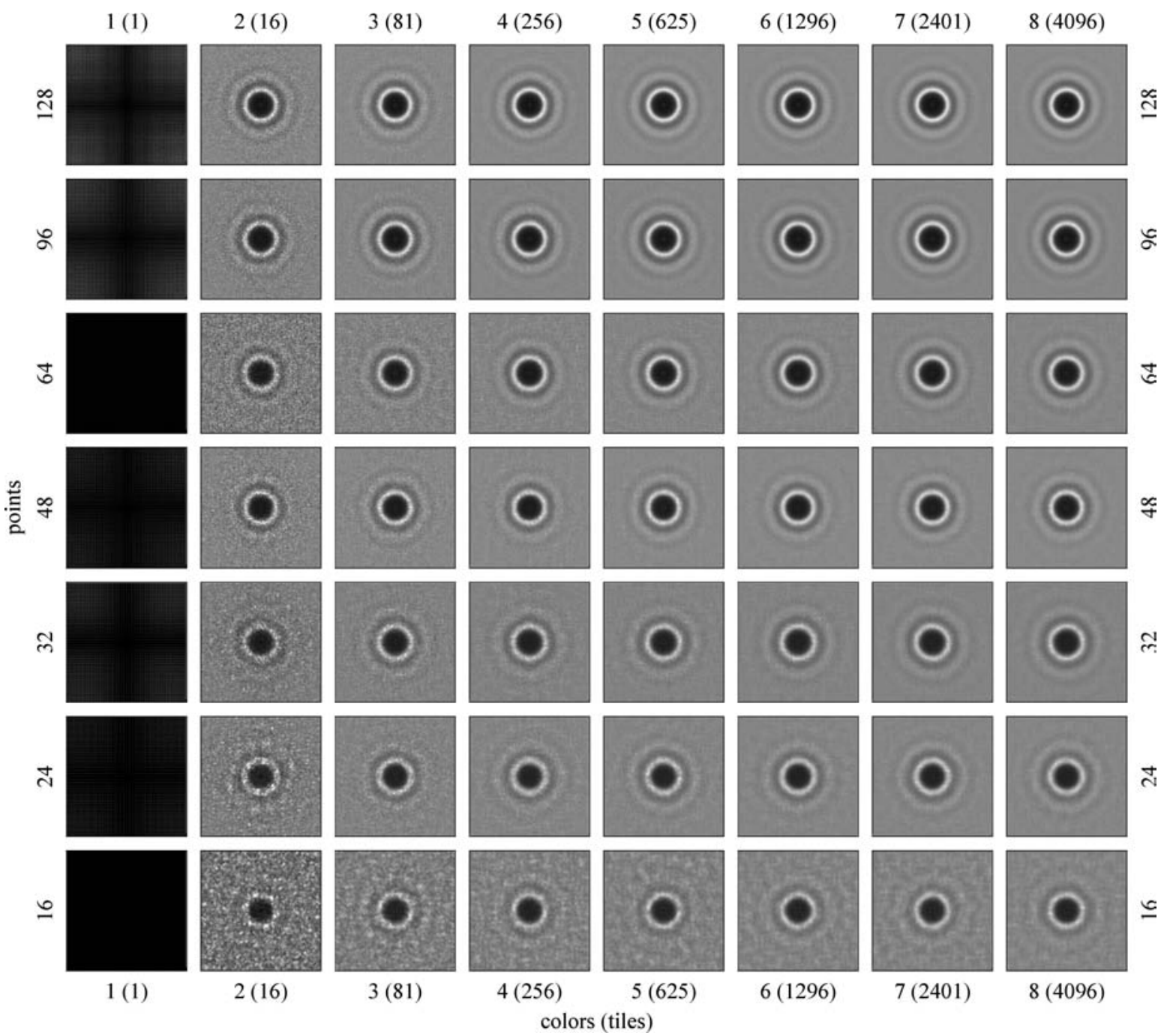

Figure 17: The power spectrum of Poisson disk distributions generated with corner-based Poisson disk tiles with a relative radius of 0.75 for corner based Poisson disk tile sets with 1,2,3,4,5,6,7 and 8 colours $(1,16,81,256,625,1296,2401$ and 4096 tiles) and 16,24,32,48,64,96 and 128 points per tile.

erating varying-density point distributions in real time. For more details, we refer to [KCODL06]. A similar technique is explored by Lagae and Dutré [LD06b].

The radius of the point distributions generated over a constant density is surprisingly low. Using the original tile set of Kopf et al., we have measured relative radii between 0.44 and less than 0.001 . Most distributions had a relative radius of less than 0.001 . The technique of Kopf $e t$ al. is therefore not suited for applications requiring a large relative radius.

Figure 20 shows the power spectrum of Poisson disk distribution generated with the approach of Kopf et al. Compared to the previous techniques, the power spectrum rather bad. The anisotropy is rather high.

The time required to generate a Poisson disk distribution of approximately 16384 tiles is 1.005 seconds.

\section{Conclusion}

Poisson disk distributions are used frequently in computer graphics. We have analysed different methods for generating Poisson disk distributions based on radial statistics and spectral properties. These two measures were chosen because they cover a wide range of applications. 

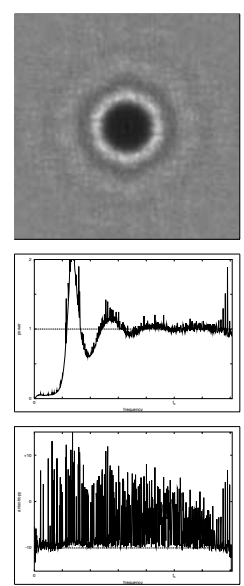

(a) 4 c., 32 p. p.t.
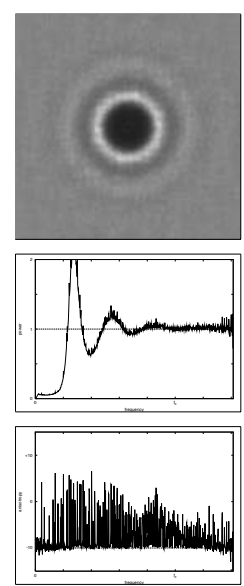

(d) 6 c., 32 p. p.t.


(g) 8 c., 32 p. p.t.


(b) 4 c., 48 p.p.t.
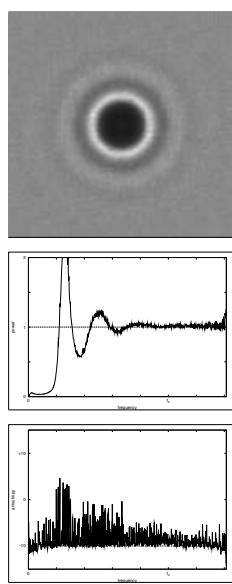

(e) 6 c., 48 p.p.t.


(h) 8 c., 48 p.p.t.
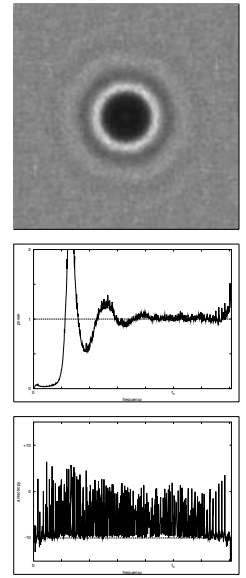

(c) 4 c., 64 p. p.t.


(f) 6 c., 64 p. p.t.
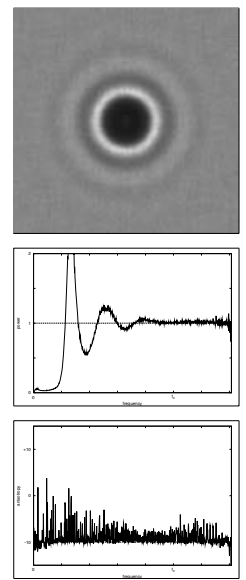

(i) 8 c., 64 p. p.t.

Figure 18: The power spectrum of Poisson disk distributions generated with corner-based Poisson disk tiles, with 4, 6 and 8 colours, 32, 48 and 64 points per tiles, and a relative radius of 0.75 .



Figure 19: The number of points in corner and edge regions versus the number of points per tile, for a relative radius of $0.70,0.75$ and 0.80 .

Dart throwing, relaxation dart throwing and Lloyd's relaxation can only be used for applications that are not interactive, and for applications that do not require large Poisson disk distributions (roughly less than 100,000 points). The accelerated dart throwing algorithms of Jones and Dunbar and Humphreys are suited for interactive applications, but they are still relatively slow compared to tile-based approaches, and if a large radius is required, must still be followed by slow Lloyd's relaxation iterations.

For real-time applications, and applications that require large Poisson disk distributions, tile-based approaches are the only option. Shade's approach has major shortcomings and should not be used. We recommend not to use tiled blue noise samples because the radius of the generated distributions is low, and because the power spectrum is relatively bad. Edgebased Poisson disk tiles are better, both in terms of radius and spectral properties. Template Poisson disk tiles are interesting from a theoretical point of view, but the toroidal tile boundary introduces too many artefacts in the power spectrum. Corner-based Poisson disk tiles have several advantages over edge based Poisson disk tiles. Corner-based Poisson disk tiles produce better power spectra even with less tiles, and allow to trade spectral quality for tile set size. Therefore, corner based Poisson disk tiles seems to be the best tile-based approach.

The spectral properties of the method of Ostromoukhov et al. and Kopf et al. are below average. The radius of the Poisson disk distributions generated with the technique of Kopf et al. is surprisingly low. The technique should not be used for generating constant density Poisson disk distributions. However, the real power of these methods is that they are capable of generating varying density point distributions. In this paper, only constant density Poisson disk distributions were investigated. 

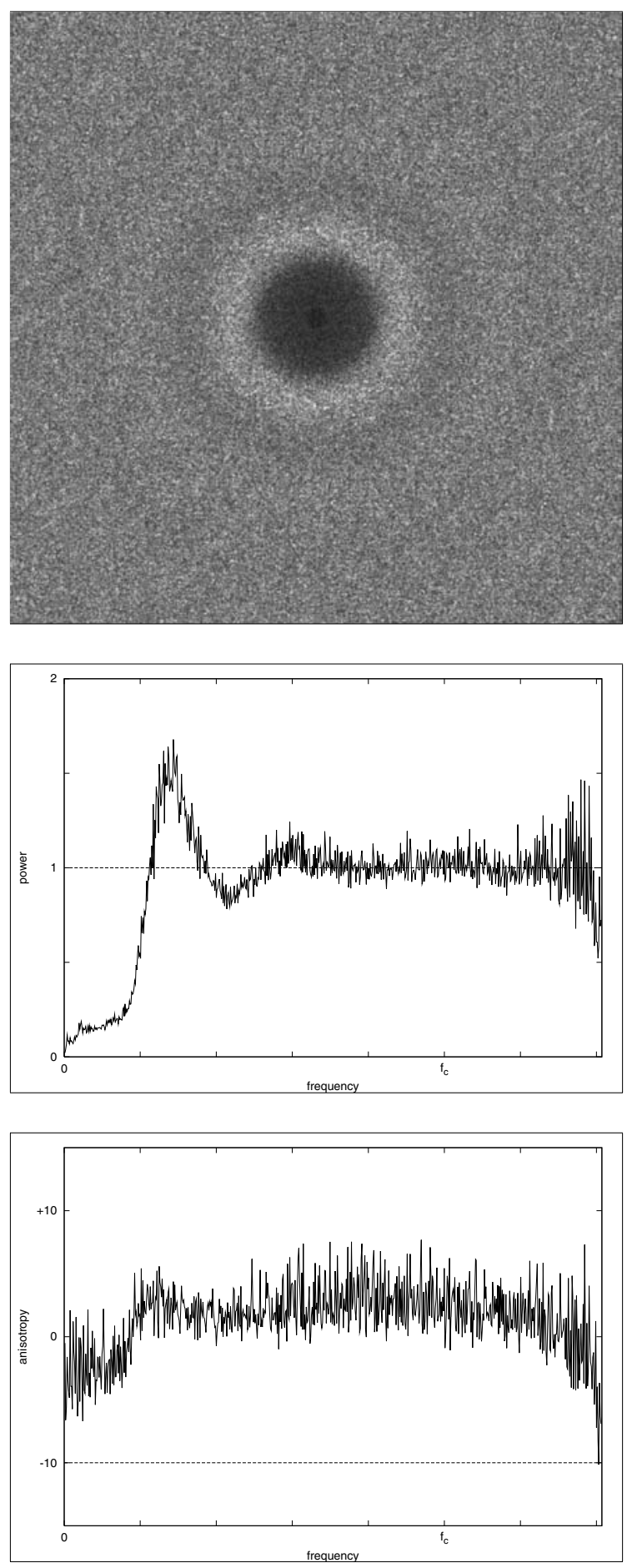

Figure 20: The power spectrum of Poisson disk distributions generated with recursive Wang tiles for real time blue noise.
We would like to note that different applications often have different requirements. For example, a large radius is very important for object distribution, while spectral properties may not be that important. For sampling, spectral properties are very important, and a slightly smaller radius will not matter that much. Radial statistics and spectral properties are two very important criteria for analysing Poisson disk distributions. However, this does not mean that for a specific application certain methods should be discarded a priori.

\section{ACKNOWLEDGMENTS}

The first author is funded as a Research Assistant by the Fund of Scientific Research - Flanders, Belgium (Aspirant F.W.O.Vlaanderen). Thanks to Stefan Hiller for sharing the data of [HDK01]. We are grateful to the authors of [ODJ04], [Jon06], [KCODL06] and [DH06] for making available example code. We would also like to thank the anonymous reviewers for their constructive remarks.

\section{References}

[Bar55] BARTLETT M.: An introduction to stochastic processes with special reference to methods and applications. Cambridge University Press, 1955.

[Coo86] CooK R. L.: Stochastic sampling in computer graphics. Computer Graphics (Proceedings of ACM SIGGRAPH 86) 5, 1 (1986), 51-72.

[Cro77] Crow F. C.: The aliasing problem in computergenerated shaded images. Communications of the ACM 20, 11 (1977), 799-805.

[CSHD03] Cohen M. F., Shade J., Hiller S., DeuSSEN O.: Wang tiles for image and texture generation. ACM Transactions on Graphics (2003), 287-294.

[DH06] Dunbar D., HumphreYs G.: A spatial data structure for fast poisson-disk sample generation. ACM Transactions on Graphics 25, 3 (2006), 503-508.

[DHL*98] Deussen O., Hanrahan P., Lintermann B., MĚch R., Pharr M., PrusinkieWICZ P.: Realistic modeling and rendering of plant ecosystems. Proceedings of ACM SIGGRAPH 1998 (1998), 275-286.

[DW85] DipPÉ M. A. Z., Wold E. H.: Antialiasing through stochastic sampling. Computer Graphics (Proceedings of ACM SIGGRAPH 85) 19, 3 (1985), 69-78.

[GS86] Grünbaum B., ShePARD G. C.: Tilings and Patterns. W. H. Freeman and Company, 1986.

[HDK01] Hiller S., Deussen O., Keller A.: Tiled blue noise samples. Proceedings of Vision Modeling Visualization 2001 (2001), 265-272. 
[Jon06] JONES T. R.: Efficient generation of Poisson-disk sampling patterns. Journal of Graphics Tools 11, 2 (2006), 27-36.

[KCODL06] Kopf J., COHEN-OR D., Deussen O., LISCHINSKI D.: Recursive wang tiles for real-time blue noise. ACM Transactions on Graphics 25, 3 (2006), 509518.

[Kla00] KLASSEN R. V.: Filtered jitter. Computer Graphics Forum 19, 4 (2000), 223-230.

[LD05a] Lagae A., Dutré P.: A procedural object distribution function. ACM Transactions on Graphics 24, 4 (2005).

[LD05b] Lagae A., Dutré P.: Template Poisson Disk Tiles. Report CW 413, Department of Computer Science, K.U.Leuven, Leuven, Belgium, May 2005.

[LD06a] Lagae A., Dutré P.: An alternative for Wang tiles: Colored edges versus coloured corners. ACM Transactions on Graphics (2006). Accepted.

[LD06b] LagaE A., DutRÉ P.: Generating WellDistributed Point Sets with a Self-Similar Hierarchical Tile. Report CW 462, Department of Computer Science, K.U.Leuven, Leuven, Belgium, August 2006.

[Llo82] LLOYD S. P.: Least squares quantization in PCM. IEEE Transactions on Information Theory 28, 2 (1982), 129-137.

[MF92] McCoOL M., FIUME E.: Hierarchical Poisson disk sampling distributions. Graphics interface '92 (1992), 94105.

[Mit87] Mitchell D. P.: Generating antialiased images at low sampling densities. Computer Graphics (Proceedings of ACM SIGGRAPH 87) 21, 4 (1987), 65-72.
[Mit91] Mitchell D. P.: Spectrally optimal sampling for distribution ray tracing. Computer Graphics (Proceedings of ACM SIGGRAPH 91) 25, 4 (1991), 157-164.

[ODJ04] Ostromoukhov V., Donohue C., Jodoin P.M.: Fast hierarchical importance sampling with blue noise properties. ACM Transactions on Graphics 23, 3 (2004), 488-495.

[Pen74] PenRose R.: The role of aesthetics in pure and applied mathematical research. Bull. Inst. Math. Applications 10 (1974), 266-271.

[SCM00] Shade J., Cohen M. F., Mitchell D. P.: Tiling Layered Depth Images. Tech. rep., University of Washington, Department of Computer Science and Engineering, 2000.

[SHS02] SECORD A., HeIdRICH W., STREIT L.: Fast primitive distribution for illustration. EGRW '02: Proceedings of the 13th Eurographics workshop on Rendering (2002), 215-226.

[Ste99] Steinhaus H.: Mathematical Snapshots. Dover Publications, Inc., Mineaola, NY, 1999.

[Uti87] UlichneY R.: Digital halftoning. MIT Press, Cambridge, MA, USA, 1987.

[Wan61] WANG H.: Proving theorems by battern recognition - II. Bell Systems Technical Journal 40 (1961), 1-42.

[Wan65] WANG H.: Games, logic and computers. Scientific American 213, 5 (1965), 98-106.

[Yel82] YELLOT J. I.: Spectral analysis of spatial sampling by photoreceptors: Topological disorder prevents aliasing. Vision Research 22 (1982), 1205-1210.

[Yel83] YELlOT J. I.: Spectral consequences of photoreceptor sampling in the rhesus retina. Science 221 (1983), 382-385. 